\title{
Outcome of Pancreaticoduodenectomy at Low-Volume Centre (Canakkale State Hospital, Turkey)
}

\author{
Nuri Emrah Goret ${ }^{1}$, Ugur Topal ${ }^{2}$ \\ ${ }^{1}$ Department of General Surgery, Health Sciences University Kartal Dr. Lutfi Kirdar \\ Training and Research Hospital, Istanbul, Turkey, ${ }^{2}$ Department of General Surgery, \\ Basaksehir Cam VE Sakura City Hospital, Istanbul, Turkey.
}

ABSTRACT

\section{BACKGROUND}

Currently, pancreaticoduodenectomy (PD) is considered a commonly performed surgery for periampullary tumours; but, it is still a high-risk surgical procedure with potential morbidity and mortality rates. Previous studies have identified a significant volume-outcome relationship for hospitals performing pancreaticoduodenectomy (PD). We intended to present the results of patients who underwent pancreatic resection with the diagnosis of malignancy in a low-volume centre.

\section{METHODS}

Patients who underwent pancreatic resection with the diagnosis of malignancy at the $2^{\text {nd }}$ stage state hospital between 2014 and 2018 were included in the study. Patients who underwent pancreatic surgery due to trauma and benign reasons were excluded from the study. Clinical data of the patients have been analysed retrospectively.

\section{RESULTS}

12 patients participated in our study. 8 patients were male. The average age was 65.75 and the oldest patient was 85 years old. The mean values of preoperative laboratory parameters were HGB gr / dl: 12.3; Albumin gr / dl 3.8 Cea ng / ml 4.08 Ca19.9 U / ml 194 Whipple procedure and the other half was applied distal pancretectomy, the mean tumor diameter was 3.67, the mean number of lymph nodes dissected was 18.1. Postoperative morbidity rate was 33.3. Pancreatic fistula, biliary fistula, wound infection and pulmonary embolism were seen in one patient each. Average hospital stay was 10.66 days. Mortality occurred in patient who had developed pulmonary embolism.

\section{CONCLUSIONS}

Pancreatic resections can be performed safely in low-volume centres, with morbidity and mortality rates comparable to high-volume centers. Patients who have difficulty in accessing high-volume academic centers can be operated in low volume centers.

\section{KEY WORDS}

Pancreatic Cancer, Low Volume Center, Morbidity
Corresponding Author:

Dr. Uğur Topal,

Department of General Surgery,

Başakşehir Çam ve Sakura City

Hospital, Istanbul, Turkey.

E-mail: sutopal2005@hotmail.com

DOI: $10.14260 / j e m d s / 2021 / 397$

How to Cite This Article:

Goret NE, Topal U. Outcome of pancreaticoduodenectomy at low-volume centre (Canakkale State hospital, Turkey). J Evolution Med Dent Sci 2021;10(26):19261930, DOI: 10.14260/jemds/2021/397

Submission 28-11-2020,

Peer Review 30-04-2021,

Acceptance 07-05-2021,

Published 28-06-2021.

Copyright (c) 2021 Nuri Emrah Goret et al.. This is an open access article distributed under Creative Commons Attribution License [Attribution 4.0 International (CC BY 4.0)] 


\section{BACKGROUND}

According to the data of the World Health Organization, periampullary region tumors, especially pancreatic cancer, rank $14^{\text {th }}$ among all cancer groups according to their spread rate and $7^{\text {th }}$ according to mortality. ${ }^{1}$ Periampullary tumors are neoplasms that occur around the ampulla of Vater. Origins; pancreas, duodenum, distal of main hepatic bile duct and ampulla vateri complex structures. Bulb carcinomas are also bulb-complex, arising from the main hepatic bile duct bifurcation distal to the pancreatic duct. Pancreaticoduodenectomy (PD) surgery was first defined by Whipple in 1935, and it is still the standard surgical treatment option for pancreatic adenocarcinoma and is potentially the only curative treatment for Periampullary tumors. Surgery can be done to only 25 - $30 \%$ of the patients due to the advanced stage of the disease at the time of diagnosis. ${ }^{2}$ In the early days, periampullary malignancy was the only indication of the PD option, but recently PD is also used in non-neoplastic cases such as chronic pancreatitis.

There are many studies which have recognised that there is a significant procedure volume - patient outcome relation for hospitals performing many surgical procedures. The biggest problem in PD surgery is the complexity of the surgical procedure and the high morbidity and mortality rates associated with the surgery. This, together with the low longterm survival rate, has led to the questioning that treatment is worse than the disease.

Significant advances in diagnostic methods, patient selection, postoperative care and many aspects of pancreatic surgery have contributed significantly to the dramatic increase in the reliability of this surgery in the modern age.These bad results are attributed to many factors related to the patient and the hospital, one of which is the number of cases per year in the center.3,4

According to NCCN 2016, the high-volume center is defined as the center where PD is applied on more than 20 cases in each year. In addition to studies in the literature stating that postoperative results will be better in high volume centers, it is also reported that pancreatic resections can be performed safely with low morbidity and operative mortality rates in lower density centers. ${ }^{5-8}$ Most data regarding centralization of PD are derived from multi-institutional comparisons, and there is a lack of studies describing the effects of increased caseload of PD within the same unit.

As a result of improved results in high volume centers, complex cancer surgery has started to be performed more and more frequently in larger centers. However, regionalization can be a significant burden for cancer patients and may delay treatment.

Many rural patients do not have the means to receive treatment in a regional referral center due to problems such as distance, travel and social support. This affects the application of optimal cancer treatment. ${ }^{9}$

Centralization of pancreatic surgery is not routine in most European countries, so data on the impact of hospital volume on the death rate are scarce. In this article, we intended to present the results of patients who underwent pancreatic surgery for malignancy in a low-volume center in the light of the literature.

\section{METHODS}

This is a case record analysis study designed retrospectively wherein patients who underwent pancreatic resection with a diagnosis of malignancy at the Canakkale state hospital for a period of 1 year between January 2014 and 2018 were included in the study. Patients who underwent pancreatic surgery due to trauma and benign reasons were excluded from the study.

From patient records, besides the demographic information of the patient such as age and gender, admission symptom American Society of Anesthesiologists (ASA) score Hgb and Albumin level Tumor markers Cea and CA19.9, surgical method applied, number of lymph nodes dissected, tumor size, postoperative complication status, postoperative hospital stay and perioperative mortality were also evaluated.

Although pre-operative imaging methods were not standard, for diagnostic and staging purposes with ultrasonography, computed tomography, magnetic resonance imaging, endoscopic retrograde cholangiopancreatography with pre-operative imaging methods, patients who were thought to be potentially resected underwent explorative laparotomy for curative resection.

PD was defined as the removal of the duodenum with the pancreatic head and neck region, the distal $1 / 3$ of the stomach, the distal caledoc and the gall bladder and the near regional lymph nodes. Pancreatic fistula was defined as the discharge of fluid with high amylase content (amylase concentration in the intraabdominal fluid more than 3 times the serum amylase concentration) from the drain for more than 3 days after surgery. Bile discharge from intra-abdominal drains was identified as biliary fistula and inflammation from the wound requiring opening of the wound was detected as wound infection.

\section{Statistical Analysis}

All statistical analysis were performed using the "Statistical Package for the Social Sciences for Windows version 21.0 (SPSS Inc.; Chicago, IL, USA) package program. Categorical measurements were summarized as numbers and percentages, while continuous measurements summarized are given as mean \pm standard deviation (minimum maximum).

\section{RESULTS}

Twelve patients participated in our study. 8 patients were male. Our average age was 65.75 and the oldest patient was 85 years old. Patients who had the most frequent ASA 2 distribution being $50 \%$. Abdominal pain was the main symptom for application. The mean values of preoperative laboratory parameters were HGB gr / dl: 12.3; Albumin gr / dl 3.8 Cea ng / ml 4.08 Ca19.9 U / ml 194 Demographic and clinical characteristics are shown in Table 1.

Half of the patients were applied Whipple procedure and the other half were applied distal resection, while adenocarcinoma being the most common tumor type in $92 \%$. The average tumor diameter was 3.67, the average number of lymph nodes dissected was 18.1 and the number of positive 
lymph nodes was 7.1. Operation and pathological data are shown in Table 2.

Our postoperative morbidity rate was 33.3. Pancreatic fistula, biliary fistula, wound infection and pulmonary embolism were seen in one patient each. Our mean hospital stay was 10.66 days. Mortality occurred in the patient who developed pulmonary embolism. Postoperative follow-up data are shown in Table 3.

\section{Ethical Statement}

The study was approved by the local Institutional Review Board. The study was analysed retrospectively in accordance with the ethical rules based on the principles of the Helsinki Declaration between 2015 and 2017.

\begin{tabular}{|c|c|c|}
\hline \multicolumn{2}{|c|}{$\begin{array}{c}\text { Variable } \\
\text { Age }+ \text { STD (min-max) }\end{array}$} & $\begin{array}{c}\mathrm{N}(\%) \\
65.75+12.5(38-85)\end{array}$ \\
\hline \multirow{2}{*}{ Gender } & Male & $8(67)$ \\
\hline & Female & $4(33)$ \\
\hline \multirow{3}{*}{ ASA score } & 1 & $4(33)$ \\
\hline & 2 & $6(50)$ \\
\hline & 3 & $2(17)$ \\
\hline \multirow{3}{*}{ Application symptom } & Abdominal Pain & $6(50)$ \\
\hline & Icterus & $4(33)$ \\
\hline & Weight loss & $2(17)$ \\
\hline \multicolumn{2}{|c|}{ Preoperative Cea ng / ml. } & $4.08+2.88(1.3-9.6)$ \\
\hline \multicolumn{2}{|c|}{ Preoperative Ca $19.9 \mathrm{U} / \mathrm{ml}$} & $194+197(23-690)$ \\
\hline \multicolumn{2}{|c|}{ Haemoglobin gr / dl } & $12.3 \pm 2.1(8.8-15.4)$ \\
\hline \multirow{2}{*}{\multicolumn{2}{|c|}{ Albumin gr / dl }} & $3.8+0.58(2.7-4.9)$ \\
\hline & & Characteristics \\
\hline
\end{tabular}

\begin{tabular}{|ccc|}
\hline \multirow{3}{*}{ Operation type } & Variable & $\mathbf{N}(\%)$ \\
& Pancreaticoduodenectomy & $6(50)$ \\
Pathological & Distal pancreatectomy & $6(50)$ \\
& Adenocarcinoma & $11(92)$ \\
& Lymphoma & $1(8)$ \\
& Tumor diameter (cm) & $3.67+2.91(1.5-$ \\
Total number of lymph nodes removed & $12)$ \\
Number of positive lymph nodes & $18.1 \pm 9.3(9-42)$ \\
\hline \multicolumn{2}{|c|}{ Table 2. Operation and Pathological Data } \\
\hline \multicolumn{2}{|c}{} \\
\hline
\end{tabular}

\begin{tabular}{|c|c|c|}
\hline \multicolumn{2}{|c|}{ Variable } & $\mathrm{N},(\%)$ \\
\hline \multirow{4}{*}{ Postoperative complication } & Pancreatic fistula & $1(8.3)$ \\
\hline & Biliary fistula & $1(8.3)$ \\
\hline & Wound infection & $1(8.3)$ \\
\hline & Pulmonary embolism & $1(8.3)$ \\
\hline \multicolumn{2}{|c|}{ Postoperative hospital stay (days) } & $10.66 \pm 3.65(4-16)$ \\
\hline \multicolumn{2}{|c|}{ 30-day mortality } & $1(8,3)$ \\
\hline \multicolumn{3}{|c|}{ Table 3. Postoperative Follow-Up } \\
\hline
\end{tabular}

\section{DISCUSSION}

The empirical relationship between high surgical volume and low postoperative mortality was defined in the study conducted by Luft et al. about 40 years ago. This view has led to the concept of performing complex surgical procedures in high-volume centers to improve postoperative outcomes. ${ }^{10}$ In some countries, for example in Germany, this operation is legally permitted in centers where at least ten pancreatic resections are performed annually. ${ }^{11}$ However, volumeoutcome relationships in pancreatic surgery still continue, although debate has diminished, as some studies have reported similar results from low-volume state hospitals to high - volume hospitals. ${ }^{12}$

The main theory used to explain the hospital volumeoutcome relationship in surgery is that repeating the same operation increases the ability of surgeons and hospitals to perform a specific procedure, and that centers' completing the learning curve is an important quality-enhancing effect, especially when it comes to complex and high-risk operations. Many factors are effective. Some of these are the surgeon's developing technical skills, being more careful in patient selection, and habits in postoperative care. ${ }^{13}$ Surgeon's volume is less emphasized in the literature, but surgeon volume is also an important part of the hospital volume effect. ${ }^{14}$

Many studies in the literature have consistent results for better outcome in pancreatic surgery with increased case volume. Shah, O. J et al. divided the hospital volume into low (less than 10 cases per year), medium (10 - 24 cases per year) and high (more than 25 cases annually) in their study. When they evaluated the PD results, the duration of the surgical procedure, blood loss, postoperative pancreatic fistula, postoperative morbidity and most importantly, postoperative mortality rates consistently decreased as the number of cases increased in the center. ${ }^{15}$

Kagedan, D. J., et al. divided the patients with PD into three groups according to the hospital volume in a cohort of 2660 patients: as low, medium and high volume hospitals. (30 and 90 day mortality was respectively ( $\% 2,9, \% 5,2)$ in lower volume hospitals, ( $\% 3,9, \% 6,3)$ in medium volume hospitals and ( $\% 1,5, \% 2,7)$ in high volume hospitals. Lowest one was in high volume hospitals $(\mathrm{P}<0.01)$. Patients operated in high and medium volume centers had lower reoperation rates compared to low volume centers ( $\% 16,7$ against $\% 10,3$, \% $10,7, P=0.0002)$. They attributed this difference to the clustering of physicians specializing in hepatobiliary surgery in high-volume hospitals. ${ }^{16}$

In the study he published the results of pancreatic resections performed in a low-volume center, Cocieru, A. found that total morbidity rate after PD was $51.5 \%$ and that it was $51 \%$ after DP. Their overall mortality rate was $0.7 \%$. Morbidity seems to be prevented from creating mortality with correct management. ${ }^{17}$ However, the delay in starting chemotherapy and the increase in hospital costs cannot be ignored due to morbidities.

In the study conducted by Vinchurkar, $\mathrm{K}$ et al. where they presented their 4 - year experience in their center where 10 cases were conducted annually, they found the mortality rate of $3.84 \%$ and the morbidity rate of $35.1 \%$. They argued in their studies that, with a well-equipped hospital infrastructure, an equipped intensive care unit and an experienced surgical team, they may not have as much effect on PD outcomes as the hospital volume. ${ }^{18}$ The study was conducted in a third world country such as India, although centralization of complex surgical procedures is suggested, but this is not always possible. Therefore, many patients are applied surgical interventions at 2nd stage State Hospitals.

Kanhere, $\mathrm{H}$. A et al. performed 53 curative pancreatic resections in the low-volume but Specialized Hepato Pancreato Biliary Units over a 14 - year period. Their mortality rate was $3.8 \%$. This was equivalent to experienced centers. The pancreatic fistula rate was $18.8 \%$. This was an acceptable rate according to the literature. The number of lymph nodes dissected was 8.7 In their studies, they emphasized the importance of specialized hepatobiliary unit such as surgeon volume and hospital volume.

In our series, the demographic and clinical characteristics of the patients were compatible with the literature. We do not think that it has a direct effect on surgical results. The cases in our series were not only patients who underwent PD, but also those who underwent DP. Considering that the results of distal 
pancreatic surgery are better, it may be a parameter that affects our results. The pancreatic fistula that developed in our series was closed with medical follow-up. Likewise, no surgical intervention was required for biliary fistula. Surgical debridement was required for the patient who developed wound site infection.

In our series we prevented patients' morbidities from evolving into mortality. The patient who developed postoperative mortality was not due to surgery. The mortality was exitus due to pulmonary embolism. The average number of lymph nodes dissected were 18 and it seemed sufficient according to the literature.

Although the dispute on volume for complex operations shifted towards surgeon's volume, Wood, T.W et al. found in their study that the results of low volume surgeons in high volume hospitals were similar to those of high volume surgeons. The conclusion we can draw here is that there is a hospital "field effect" and that the hospital volume remains important. 12

Enomoto, L.M et al. found their series to have a three-fold increased mortality rate in patients operated by low-volume surgeons in low-volume hospitals compared to patients operated by high-volume surgeons in high-volume hospitals. In the same study, they found a cost difference of approximately $\$ 12,000$ between these two groups. In their work, surgeons with high volumes were generally working in high volume hospitals, which was another factor that contributed to this striking effect. 5

Another advantage of high volume hospitals is the advanced intensive care conditions and the presence of interventional radiology teams and developing radiological imaging methods. In this way, complications were detected early and less invasive treatment was possible. Complications were prevented from evolving to death. ${ }^{18}$

In another study in the literature, Stella, $M$ et al. showed that the results of pancreatic resection performed by surgical specialists from high-volume centers in low - volume hospitals can achieve surgical results similar to high - volume centers. ${ }^{19}$

\section{CONCLUSIONS}

From these studies, we can conclude that the surgical experience is as important as the hospital volume. Surgical quality may increase with the transfer of surgeons who gain surgical experience in high volume hospitals to low volume centers. Surgical results seem to be influenced by hospital volume, surgeon volume, and many parameters related to the patient. However, complex operations such as pancreatic resections can be performed with low mortality and acceptable morbidity rates in low-volume centers. Patients who have difficulties in accessing high-volume academic centers can be operated in low volume centers.

\section{Limitations}

Patients had no long-term oncologic results and the data was retrospective in nature.

Data sharing statement provided by the authors is available with the full text of this article at jemds.com.

Financial or other competing interests: None.
Disclosure forms provided by the authors are available with the full text of this article at jemds.com.

\section{REFERENCES}

[1] Bray F, Ferlay J, Soerjomataram I, et al.. Global cancer statistics 2018: GLOBOCAN estimates of incidence and mortality worldwide for 36 cancers in 185 countries. CA Cancer J Clin 2018;68(6):394-424.

[2] Pallisera A, Morales R, Ramia JM. Tricks and tips in pancreatoduodenectomy. World J Gastrointest Oncol 2014;6(9):344-50.

[3] Macedo FIB, Jayanthi P, Mowzoon M, et al.. The impact of surgeon volume on outcomes after pancreaticoduodenectomy: a meta-analysis. J Gastrointest Surg 2017;21(10):1723-31.

[4] Newhook TE, LaPar DJ, Lindberg JM, et al.. Morbidity and mortality of pancreaticoduodenectomy for benign and premalignant pancreatic neoplasms. J Gastrointest Surg 2015;19(6):1072-7.

[5] Enomoto LM, Gusani NJ, Dillon PW, et al.. Impact of surgeon and hospital volume on mortality, length of stay and cost of pancreaticoduodenectomy. J Gastrointest Surg 2014;18(4):690-700.

[6] El Amrani M, Clement G, Lenne X, et al.. Failure-to-rescue in patients undergoing pancreatectomy: is hospital volume a standard for quality improvement programs? Nationwide analysis of 12,333 patients. Ann Surg 2018;268(5):799-807.

[7] Kanhere HA, Trochsler MI, Kanhere MH, et al.. Pancreaticoduodenectomy: outcomes in a low-volume, specialised hepato pancreato biliary unit. World J Surg 2014;38(6):1484-90.

[8] Swan RZ, Lau KN, Sindram D, et al.. Pancreatic resection in a large tertiary care community-based hospital: building a successful pancreatic surgery program. Surg Oncol Clin N Am 2011;20(3):487-500.

[9] Sabesan A, Gough BL, Anderson C, et al.. High volume pancreaticoduodenectomy performed at an academic community cancer center. Am J Surg 2019;218(2):349-54.

[10] Luft HS, Bunker JP, Enthoven AC. Should operations be regionalized? The empirical relation between surgical volume and mortality. N Engl J Med 1979;301(25):13649.

[11] Alsfasser G, Kittner J, Eisold S, et al.. Volume-outcome relationship in pancreatic surgery: the situation in Germany. Surgery 2012;152(3 Suppl 1):S50-5.

[12] Wood TW, Ross SB, Bowman TA, et al.. High-volume hospitals with high-volume and low-volume surgeons: is there a field effect for pancreaticoduodenectomy? Am Surg 2016;82(5):407-11.

[13] Ansari D, Williamsson C, Tingstedt B, et al. Pancreaticoduodenectomy--the transition from a low- to a high-volume center. Scand J Gastroenterol 2014;49(4):481-4.

[14] Eppsteiner RW, Csikesz NG, McPhee JT, et al.. Surgeon volume impacts hospital mortality for pancreatic resection. Ann Surg 2009;249(4):635-40.

[15] Shah OJ, Singh M, Lattoo MR. Pancreaticoduodenectomy: a study from India on the impact of evolution from a low 
to a high volume unit. World J Gastrointest Surg 2016;8(8):583-9.

[16] Kagedan DJ, Goyert N, Li Q, et al.. The impact of increasing hospital volume on 90-day postoperative outcomes following pancreaticoduodenectomy. J Gastrointest Surg 2017;21:506-15.
[17] Cocieru A, Saldinger PF. HPB surgery can be safely performed in a community teaching hospital. J Gastrointest Surg 2010;14(11):1853-7.

[18] Vinchurkar K, Pattanshetti VM, Togale M, et al.. Outcome of pancreaticoduodenectomy at low-volume centre in tier-II city of India. Indian J Surg Oncol 2018;9(2):220-4.

[19] Amini N, Spolverato G, Kim Y, et al.. Trends in hospital volume and failure to rescue for pancreatic surgery. J Gastrointest Surg 2015;19(9):1581-92. 